\title{
Colored Simultaneous Geometric Embeddings and Universal Pointsets
}

\author{
Ulrik Brandes - Cesim Erten - Alejandro Estrella-Balderrama • \\ J. Joseph Fowler · Fabrizio Frati · Markus Geyer · Carsten Gutwenger • \\ Seok-Hee Hong • Michael Kaufmann • Stephen G. Kobourov • Giuseppe Liotta • \\ Petra Mutzel · Antonios Symvonis
}

\begin{abstract}
Universal pointsets can be used for visualizing multiple relationships on the same set of objects or for visualizing dynamic graph processes. In simultaneous geometric embeddings, the same point in the plane is used to represent the same object as a way to preserve the viewer's mental map. In colored simultaneous embeddings this restriction is relaxed, by allowing a given object to map to a subset of points in the plane. Specifically, consider a set of graphs on the same set of $n$ vertices partitioned into $k$ colors. Finding a corresponding set of $k$-colored points in the plane such that each vertex is mapped to a point of the same color so as to allow a straightline plane drawing of each graph is the problem of colored simultaneous geometric embedding.
\end{abstract}

Work on this paper began at the BICI Workshop on Graph Drawing, held in Bertinoro, Italy in March 2006.

U. Brandes

Department of Computer \& Information Science, University of Konstanz, Konstanz, Germany

e-mail: ulrik.brandes@uni-konstanz.de

C. Erten

Department of Computer Science, Isik University, Istanbul, Turkey

e-mail: cesim@isikun.edu.tr

A. Estrella-Balderrama · J.J. Fowler $(\bowtie) \cdot$ S.G. Kobourov

Department of Computer Science, University of Arizona, Tucson, AZ, USA

e-mail: fowler@email.arizona.edu

A. Estrella-Balderrama

e-mail: alexeb@email.arizona.edu

S.G. Kobourov

e-mail: kobourov@cs.arizona.edu

F. Frati

Department of Computer Science, University of Roma Tre, Rome, Italy

e-mail: frati@dia.uniroma3.it 
For $n$-vertex paths, we show that there exist universal pointsets of size $n$, colored with two or three colors. We use this result to construct colored simultaneous geometric embeddings for a 2-colored tree together with any number of 2-colored paths, and more generally, a 2-colored outerplanar graph together with any number of 2-colored paths. For $n$-vertex trees, we construct small near-universal pointsets for 3 -colored caterpillars of size $n, 3$-colored radius- 2 stars of size $n+3$, and 2-colored spiders of size $n$. For $n$-vertex outerplanar graphs, we show that these same universal pointsets also suffice for 3-colored $K_{3}$-caterpillars, 3-colored $K_{3}$-stars, and 2-colored fans, respectively. We also present several negative results, showing that there exist a 2-colored planar graph and pseudo-forest, three 3-colored outerplanar graphs, four 4-colored pseudo-forests, three 5-colored pseudo-forests, five 5-colored paths, two 6-colored biconnected outerplanar graphs, three 6-colored cycles, four 6-colored paths, and three 9-colored paths that cannot be simultaneously embedded.

Keywords Simultaneous embedding · Simultaneous geometric embedding · Colored simultaneous embedding · Universal pointsets · Graph drawing

\section{Introduction}

Visualizing multiple related graphs is useful in many applications, such as software engineering, networking, and bioinformatics. Consider the case where a pair of related graphs is given and the goal is to visualize them so as to compare and contrast the graphs, such as two evolutionary trees on the same set of species obtained by different algorithms. When visually examining relational information, such as a graph structure, viewers construct an internal model called the mental map, for example,

\footnotetext{
M. Geyer - M. Kaufmann

Wilhelm-Schickard-Institute of Computer Science, University of Tübingen, Tübingen, Germany M. Geyer

e-mail: geyer@informatik.uni-tuebingen.de

M. Kaufmann

e-mail: mk@informatik.uni-tuebingen.de

C. Gutwenger - P. Mutzel

Department of Computer Science, University of Dortmund, Dortmund, Germany

C. Gutwenger

e-mail: carsten.gutwenger@cs.uni-dortmund.de

P. Mutzel

e-mail: petra.mutzel@cs.uni-dortmund.de

S.-.H. Hong

NICTA Ltd. and School of Information Technologies, University of Sydney, Sydney, Australia e-mail: shhong@it.usyd.edu.au

G. Liotta

School of Computing, University of Perugia, Perugia, Italy e-mail: liotta@diei.unipg.it
}

A. Symvonis

School of Applied Mathematics \& Physical Sciences, National Technical University of Athens, Athens, Greece

e-mail: symvonis@math.ntua.gr 
by using the positions of the vertices relative to each other. When viewing multiple graphs the viewer has to reconstruct this mental map after examining each graph. A common goal is to aid the viewer in this reconstruction while providing a readable drawing for each graph individually. Simultaneous embeddings [5] aid in visualizing multiple relationships between the same set of objects by keeping common vertices and edges of these graphs in the same positions.

A simultaneous geometric embedding is a generalization of the traditional planar graph embedding problem, where we look for a common embedding of multiple graphs defined on the same vertex set. We omit the "geometric" clarification in the rest of the paper as we only consider straight-line drawings. There are two main variations of the problem. In simultaneous embedding with mapping it is assumed that a mapping between the vertices of the graphs is given as part of the input: i.e., if $u$ and $v$ are two distinct vertices of different graphs and there is a mapping from $u$ to $v$, then in a simultaneous embedding of these graphs $u$ and $v$ are placed at the same point in the plane. In simultaneous embedding without mapping the simultaneous embedding consists of plane drawings for each of the given graphs on the same set of points, where any one vertex can be placed at any one of the points in the point set.

Restricted subclasses of planar graphs, such as pairs of paths, pairs of cycles, and pairs of caterpillars, admit a simultaneous embedding with mapping as shown by Brass et al. [5]. In contrast, there exist pairs of outerplanar graphs, triples of paths, and even (planar graph, path) pairs that do not always admit a simultaneous embedding with mapping [5, 15]. Recently, Kaufmann et al. [19] constructed an example of two trees that do not allow a simultaneous embedding. While any planar graph can be simultaneously embedded without mapping with any number of outerplanar graphs, it is unknown whether the same can be done for any pair of planar graphs [5].

Simultaneous embedding problems are related to universal pointsets problems, where the goal is to find a pointset $P$ in the Euclidean plane that allows for any number of graphs of a given class to be drawn with straight-line edges and no crossings on $P$. In 1986, Rosenstiehl and Tarjan [21] asked whether there exists a universal pointset of size $n$ for all $n$-vertex planar graphs. In 1990, de Fraysseix et al. [8] presented a set of $n$-vertex planar graphs requiring a pointset of size $\Omega(n+\sqrt{n})$. However, for restricted classes of $n$-vertex planar graphs universal pointsets of size $n$ exist. Gritzman et al. [17] showed that a set of $n$ points in general position is a universal pointset for trees and outerplanar graphs for which Bose [4] described efficient drawing algorithms.

Colored simultaneous embeddings allow us to generalize the problems above so that the versions with and without mappings become special cases. Formally, the problem of colored simultaneous embedding (CSE) is defined as follows. The input is a set of planar graphs $G_{1}=\left(V, E_{1}\right), G_{2}=\left(V, E_{2}\right), \ldots, G_{r}=\left(V, E_{r}\right)$ on the same vertex set $V$ and a partition of $V$ into $k$ classes, which we refer to as colors. The goal is to find plane drawings $D_{i}$ of $G_{i}$ using the same $|V|$ points in the plane for all $i=1, \ldots, r$, where vertices mapped to the same point are required to be of the same color. The output is a set of points $|P| \geq n$ that are also strictly partitioned into $k$ colors $^{1}$ with a fixed embedding in the Euclidean plane such that each graph $G_{j}$

\footnotetext{
${ }^{1}$ Technically we have "universal" pointsets only when $|P|=n$; when $|P|$ is not much larger than $n$ we have what de Fraysseix et al. [7] call "small supporting sets". In this paper, we only deal with pointsets
} 


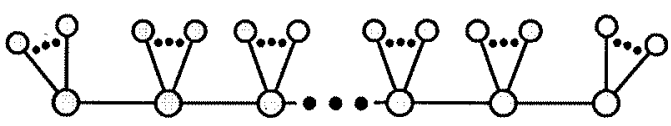

(a)

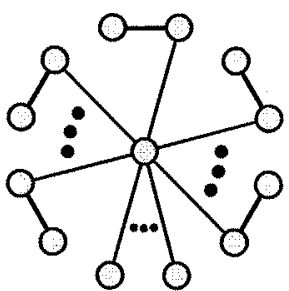

(b)

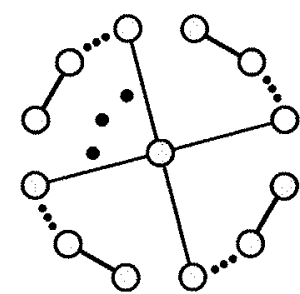

(c)

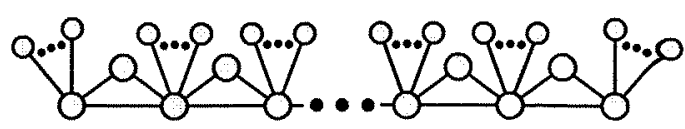

(d)

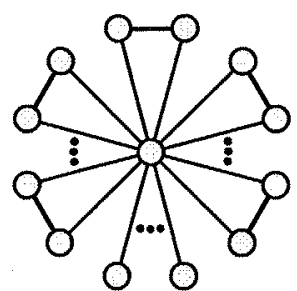

(e)

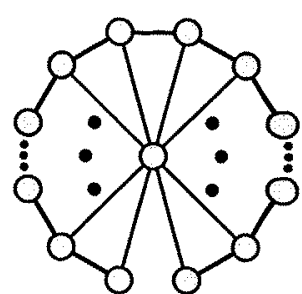

(f)

Fig. 1 Three classes of trees, (a) caterpillars (removal of all degree-1 vertices yields a path), (b) radius-2 stars (stars, $K_{1, k}$, with each edge subdivided at most once), and (c) spiders (stars with each edge subdivided arbitrarily); and three classes of outerplanar graphs, (d) $K_{3}$-caterpillars (formed from caterpillars by adding edges $(u, w)$ and $(w, v)$ to any spine edge $(u, v)$ ), (e) $K_{3}$-stars (formed from stars by adding an edge to connect any pairs of leaves), (f) fans (biconnected outerplanar graphs where all chords are incident to a common vertex)

for $j \in[1, \ldots, r]$ has a straight-line planar drawing where each vertex of color $c_{i}$ for $i \in[1, \ldots, k]$ is placed on exactly one point of $P$ also of color $c_{i}$. We call such graphs $k$-colored graphs.

Simultaneous embeddings with and without mapping then corresponds to colored simultaneous embeddings with $k=n$ and $k=1$, respectively. Thus, when a set of input graphs allows for a simultaneous embedding without mapping but does not allow for a simultaneous embedding with mapping, there must be a threshold for the number of colors beyond which the graphs can no longer be embedded simultaneously.

Colored simultaneous embeddings provide a way to obtain near-simultaneous embeddings, where we place corresponding vertices nearly, but not necessarily exactly, at the same locations. Relaxing the constraint on the size of the pointset allows for a way to more easily obtain near-simultaneous embeddings, where we attempt to place corresponding vertices relatively close to one another in each drawing. For example, if each cluster of points in the plane has a distinct color, then even if a red vertex $v$ placed at a red point $p$ in $G_{1}$ has moved to another red point $q$ in $G_{2}$, the movement is limited to the area covered by the red points. This has applications in visualizing dynamic graphs, where the viewer's mental map is preserved by limiting the movement of the vertices $[10,16]$.

In this paper, we consider various universal pointsets and colored simultaneous embeddings of paths, trees, pseudo-forests (graphs with at most one cycle), and outerplanar graphs. We also give universal pointsets for three classes of trees-caterpillars, radius-2 stars, and spiders-and three classes of outerplanar graphs $-K_{3}$-caterpillars, $K_{3}$-stars, and fans; see Fig. 1 . Our contributions consist of the following results:

(1) There exist universal pointsets of size $n$ for 2-colored paths and spiders as well as 3 -colored paths, caterpillars, and $K_{3}$-caterpillars.

$|P|=n+c$, where $c$ is a small constant less than 4 , and therefore, we still refer to these near-universal pointsets as universal. 
(2) There exist near-universal pointsets for 2-colored fans of size $n+1$ and for 3 -colored radius- 2 stars and $K_{3}$-stars of size $n+3$.

(3) A 2-colored tree or a 2-colored outerplanar graph and any number of 2-colored paths can be simultaneously embedded.

(4) There exist a 2-colored planar graph and pseudo-forest, three 3-colored outerplanar graphs, four 4-colored pseudo-forests, three 5-colored pseudo-forests, five 5-colored paths, two 6-colored biconnected outerplanar graphs, three 6-colored cycles, four 6-colored paths, and three 9-colored paths that cannot be simultaneously embedded.

\section{Universal Pointsets for Paths}

In this section, we consider universal pointsets for paths with at least two colors.

\subsection{Paths on Two Colors}

We define an $(r, b)$-separable pointset $P$ in the plane to be a set of $r$ red and $b$ blue distinct points in general position such that there exists a line $\ell$ defining two open half-planes with all red points strictly contained in one open half-plane and all blue points strictly contained in the other open half-plane. The next lemma extends a result of Abellanas et al. [1] on proper 2-colorings of paths, which will form the basis of a universal pointset for 2-colored paths.

Lemma 1 Let vertex set $V$ consist of $r$ red and $b$ blue vertices of a 2-colored path $G$. Furthermore, let $P$ be an $(r, b)$-separable pointset in the plane separated by some line $\ell$. Then there exists a planar straight-line embedding of the path $G$ onto $P$.

Proof Via a simple translation and rotation, we may assume without loss of generality that $P$ is an $(r, b)$-separable set with $\ell$ as the $y$-axis such that the red points are in the left half-plane.

We enumerate the vertices of the path $G$ from one endpoint $v_{1}$ to the other endpoint $v_{n}$ where $v_{j}$ is considered to be the $j$ th vertex along the path for $j \in\{1, \ldots, n\}$. Our embedding algorithm requires that the colors of the last two vertices of the path $G$ differ. To ensure that this is always the case, we add one vertex $v_{n+1}$ of the opposite color of $v_{n}$ to $V$ and one edge $\left(v_{n}, v_{n+1}\right)$ to the end of the path $G$. We also add an additional point $p_{n+1}$ of the same color as $v_{n+1}$ to $P$. We place $p_{n+1}$ at $\left(-\varepsilon, y_{\max }+1\right)$ if $v_{n+1}$ is colored red or at $\left(\varepsilon, y_{\max }+1\right)$ if $v_{n+1}$ is colored blue, where $\varepsilon=\frac{1}{2} \min \left\{\left|x_{p}\right|: p \in P\right\}, y_{\max }=\max \left\{y_{p}: p \in P\right\}$, and $x_{p}$ and $y_{p}$ are the $x$-coordinate and $y$-coordinate of point $p$, respectively. As a result, $p_{n+1}$ lies closer to $\ell$ than any other point in $P$ and also lies above every other point in $P$. At the end of the embedding algorithm, we delete the vertex $v_{n+1}$ from $V$ along with the corresponding point $p_{n+1}$ from $P$.

We define the upward envelope $E$ of a pointset $P$ to be the intersection of the following half-planes:

(a) the right half-plane of the vertical line passing through the leftmost point of $P$,

(b) the left half-plane of the vertical line passing through the rightmost point of $P$, 


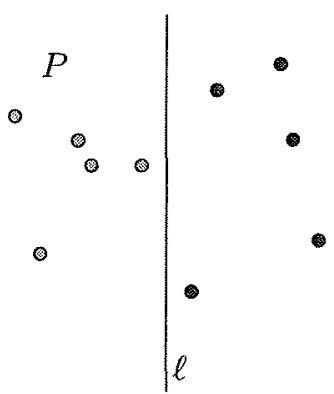

(a)

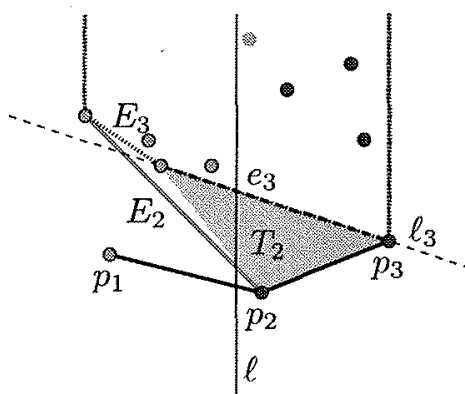

(d)

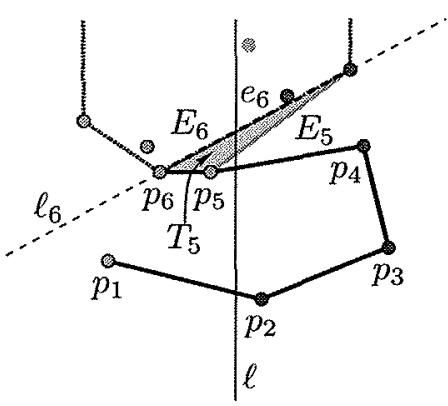

(g)

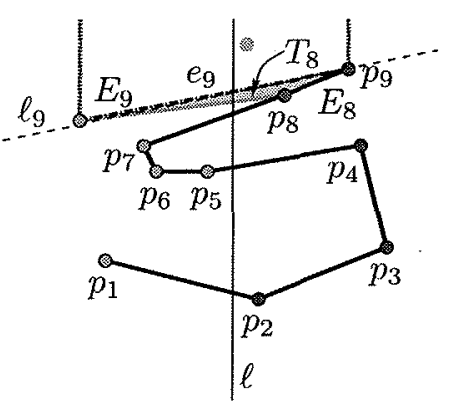

(j)

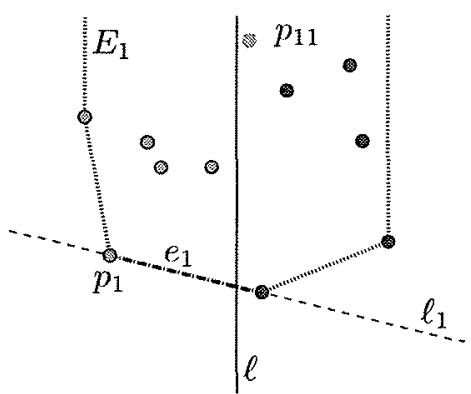

(b)

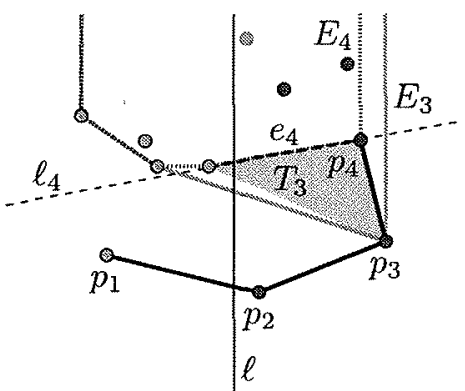

(e)

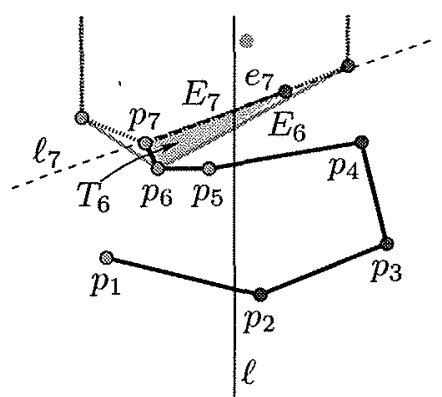

(h)

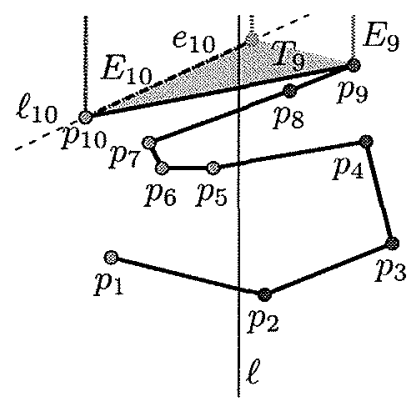

(k)

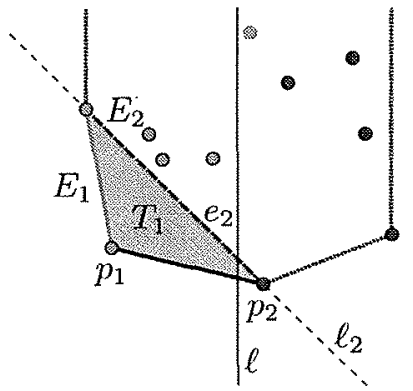

(c)

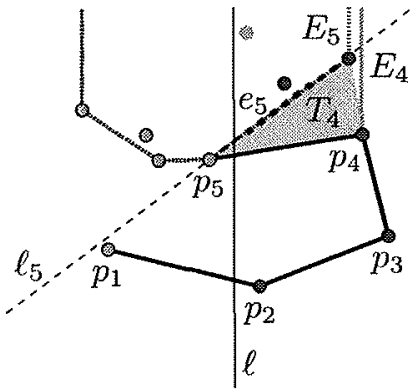

(f)

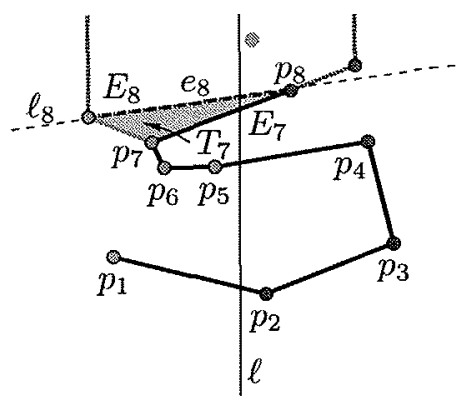

(i)

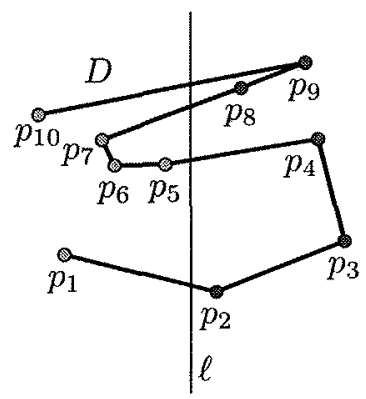

(I)

Fig. 2 Step-by-step example of embedding a 2-colored path $G$ onto a 2-colored pointset $P$ of size 10 in (a) to obtain a crossing-free drawing $D$ of $G$ in the final step (l)

(c) and the set of upper half-planes whose boundary lines pass through the edges of the polygonal chain along the lower convex hull of $P$.

For example, the pointset $P$ in Fig. 2(a) has the upward envelope $E_{1}$ shown in Fig. 2(b). Using this definition, we construct a sequence of upward envelopes 
$E_{1}, \ldots, E_{n}$ based upon a sequence of edges $e_{1}, \ldots, e_{n}$, where edge $e_{j}$ is an edge along the boundary of $E_{j}$ for $j \in\{1, \ldots, n\}$. We wish for each edge $e_{j}$ to have endpoints that correspond to a (red, blue) pair of points in $P$. We observe that each upward envelope can have at most one such edge, and if so, such an edge must intersect the $y$-axis, the separation line $\ell$ of $P$. Moreover, such an edge $e_{j}$ exists along $E_{j}$ provided that the upward envelope contains at least one red and at least one blue point.

We first let $E_{1}$ denote the upward envelope of the points $P_{1}=P$. Since $P$ must have at least one point of each color by the addition of $p_{n+1}, E_{1}$ must have an edge $e_{1}$ that crosses the $y$-axis whose endpoints have distinct colors in $P_{1}$. Suppose that $E_{j}$ contains a subset of points $P_{j}$ of $P$ such that $\left|P_{j}\right|>2$ and there exists an edge $e_{j}$ along the boundary of $E_{j}$ whose endpoints have distinct colors in $P_{j}$ for some $j \in\{1, \ldots, n-1\}$. Let $E_{j+1}$ then denote the upward envelope of the points of $P_{j+1}=$ $P_{j} \backslash\left\{p_{j}\right\}$ where $p_{j}$ is the point in $P_{j}$ that corresponds to the endpoint of $e_{j}$ matching the color of $v_{j}$.

This gives a sequence of upward envelopes $E_{1}, \ldots, E_{k}$ for some $k \in\{1, \ldots, n\}$. We contend that $k=n$. Suppose instead that $k<n$. Either $\left|P_{k-1}\right| \leq 2$ or $P_{k-1}$ only contains points of the same color. Since $|P|=\left|P_{1}\right|=n+1$ and $\left|P_{k}\right|=n+2-k$, this implies that $\left|P_{k-1}\right|=n+3-k$. If $\left|P_{k-1}\right| \leq 2$, then $n+3-k \leq 2$, or $k \geq n+1$, or $k>n$, which contradicts $k<n$. Next, observe that the point $p_{n+1}$ must be contained in every upward envelope $E_{j}$ for $j \in\{1, \ldots, k\}$ since $p_{n+1}$ lies closer to the $y$-axis than any other point in $P$ and has a $y$-coordinate strictly greater than the $y$-coordinate of any other point in $P$. Thus, for $P_{k-1}$ to only contain points of the same color, all the points in $P_{k-1}$ must have the same color as $v_{n+1}$. However, there must be a point remaining in $P_{k-1}$ that can match the color of $v_{n}$, which differs from the color $v_{n+1}$ giving a contradiction.

Let $D_{j}$ denote the drawing after embedding the vertices $v_{1}, \ldots, v_{j}$ onto the points $p_{1}, \ldots, p_{j}$, respectively, and for $j \in\{2, \ldots, n\}$, the points $p_{i-1}$ to $p_{i}$ connected by the line segment $\overline{p_{i-1} p_{i}}$ that corresponds to the edge $\left(v_{i-1}, v_{i}\right)$ in $G$. We claim that $D=D_{n}$ is a crossing-free drawing of the path $G$.

To show this, we first observe that since the points of $P$ are in general position, the convex region $E_{j}$ must strictly contain the convex region $E_{k}$ for $1 \leq j<k \leq n$, i.e., the region $E_{j}-E_{k}$ has a non-zero area, so that $E_{1}, \ldots, E_{n}$ forms a sequence of strictly nested upward envelopes. Hence, the convex region of $E_{1}$ that must contain all of the drawing $D$ is partitioned into the $n$ open regions, $E_{1}-E_{2}, \ldots, E_{n-1}-E_{n}, E_{n}$.

For $j \in\{1, \ldots, n\}$, let $\ell_{j}$ denote the line that passes though the edge $e_{j}$, and let $H_{j}$ denote the upper half-plane bounded by $\ell_{j}$ containing the convex region $E_{j}$. Let $T_{i}$ denote the triangle given by the point $p_{i}$ and the two endpoints of the edge $e_{i+1}$ for $i \in\{1, \ldots, n-1\}$. Observe that the edge $e_{i+1}$ of $E_{i+1}$ must lie in the half-plane $H_{i}$. Hence, $T_{i}$ is contained in the region $E_{i}$. The point $p_{i}$ cannot lie in the region $E_{i+1}$ by definition. The line segment $\overline{p_{i} p_{i+1}}$ also cannot intersect the boundary of $E_{i+1}$ (except at $p_{i+1}$ ), since otherwise $p_{i}$ would lie in $H_{i+1}$, and $E_{i+1}$ would then contain $p_{i}$ giving a contradiction.

Thus, $T_{i}$ must be contained in the region $E_{i}-E_{i+1}$. Since the line segment $\overline{p_{i} p_{i+1}}$ is one of the sides of the $T_{i}$, and the convex region $E_{1}$ is partitioned into the $n$ regions, $E_{1}-E_{2}, \ldots, E_{n-1}-E_{n}, E_{n}$, the drawing $D$ cannot self intersect. 


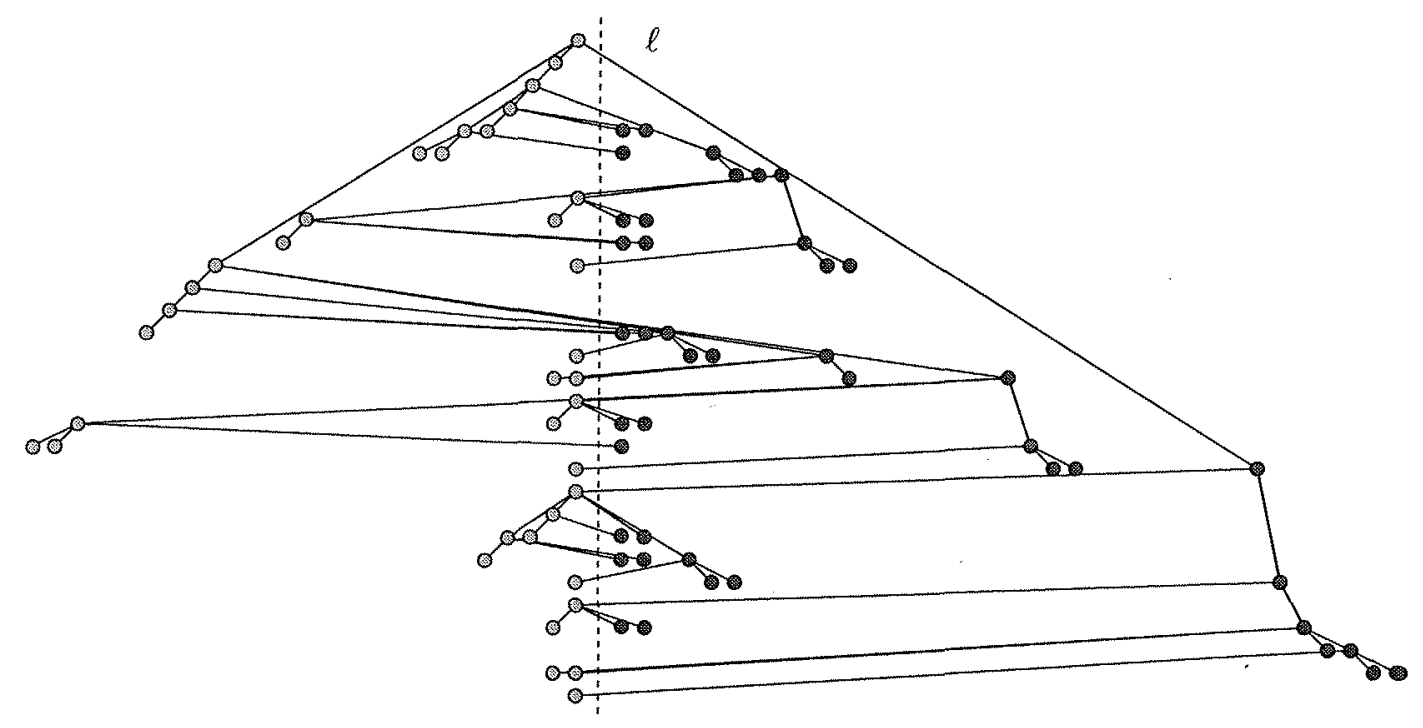

Fig. 3 Example embedding of a 2-colored tree so that the red and blue vertices lie to the left and right of line $\ell$, respectively, to obtain an $(r, b)$-separable pointset

Clearly by Lemma 1 , any $(r, b)$-separable pointset is universal for corresponding 2-colored paths, which yields our next theorem:

Theorem 2 Any $(r, b)$-separable pointset $P$ of size $n=r+b$ forms a universal pointset onto which any number of 2-colored paths with $r$ red vertices and $b$ blue vertices can be simultaneously embedded.

\subsection{A Tree and Paths on Two Colors}

We first show that it is always possible to draw a 2-colored tree so that the two colors are separated by a line $\ell$. Unlike before in which we first specify an $(r, b)$-separable pointset, and then show how to embed the given graph, we instead use the coloring of the given tree $T$ to find an $(r, b)$-separable pointset $P$ onto which to embed $T$.

Lemma 3 For any given n-vertex 2-colored tree $T$ with $r$ red vertices and $b$ blue vertices, there exists an $(r, b)$-separable pointset $P$ of size $n=r+b$ onto which $T$ can be embedded with straight-line edges and no crossings.

Proof We denote the $y$-axis as vertical line $\ell$ and assign the left half-plane bounded by $\ell$ to the color red and the right half-plane bounded by $\ell$ to the color blue. We place an arbitrary root node at $(-1,0)$ if the node is colored red and at $(1,0)$ if the node is colored blue. We process the tree starting from the root node in DFS (depth-first search) order. Given a choice of two children $u$ and $w$ of different colors of a given parent node $v$, we always process the red child before the blue child. Suppose the child node $u$ of $v$ is colored red. We place $u$ at $\left(x_{\min }-d, y_{\min }\right)$ where $x_{\min }$ and $y_{\min }$ are the minimum $x$-coordinate and $y$-coordinate, respectively, of any subtree of any sibling of $u$ placed so far, and $d$ is a positive integer sufficiently large such that the line segment connecting $v$ to $u$ does not cross any of the subtrees of $v$ placed so far. If instead $u$ is colored blue, then we place $u$ at $\left(x_{\max }+d, y_{\min }\right)$ where $x_{\max }$ is the maximum $x$-coordinate of any subtree of any sibling of $u$ placed so far. Figure 3 
gives an example of this algorithm. Given that a red (or blue) child being placed can always be shifted sufficiently far to the left (or right), no crossings will be introduced, giving a planar embedding of $T$ such that the red and blue vertices of $T$ are to the left and right of $\ell$, respectively. Finally, we perturb the positions of the vertices until they are in general position. This can be done without introducing crossings as shown in [5], which gives our $(r, b)$-separable pointset $P$.

Combining Lemma 3 and Theorem 2, we can embed a given 2-colored tree $T$ onto some $(r, b)$ separable pointset $P$, which yields the next theorem:

Theorem 4 For every 2-colored $n$-vertex tree $T$ there exists a 2-colored universal pointset $P$ of size $n$ onto which $T$ can be simultaneously embedded with any number of correspondingly 2-colored n-vertex paths.

\subsection{A Planar Graph and Paths on Two Colors}

The previous section gave a simple algorithm to construct an $(r, b)$-separable pointset onto which to embed a given 2-colored tree. In this section, we present a characterization of the class of 2-colored planar graphs for which such $(r, b)$-separable pointsets exist. We shall see that in order to simultaneously embed a 2-colored planar graph $G$ with any number of 2-colored paths, it suffices to find a plane drawing of $G$ where the red and blue vertex sets, $V_{1}$ and $V_{2}$, are separated by a line $\ell$. Once perturbed into general position, this gives an $(r, b)$-separable pointset $P$ onto which $G$ can be embedded provided $\left|V_{1}\right|=r$ and $\left|V_{2}\right|=b$. Let $G_{1}$ and $G_{2}$ be the two subgraphs induced by the vertex sets $V_{1}$ and $V_{2}$. We call such a partition a bipartition, and call the edges with endpoints from both graphs bipartition edges.

We make extensive use of the characterization and the embedding algorithm for HH layouts by Biedl et al. [3]. The problem of drawing planar partitions was introduced by Biedl [2]. An HH layout is a drawing of a planar bipartition without crossings (but not necessarily using straight-line edges) such that the two vertex sets are separated by a horizontal line. We also rely on a technique for computing upward planar straight-line drawings by Di Battista and Tamassia [9]. We begin with an apparent necessary condition for a planar bipartition to be drawn as a $\mathrm{HH}$ layout.

Lemma 5 [3] A planar bipartition of a planar graph $G$ has a HH layout only if the subgraph $H_{D}$ of the dual graph of $G$ induced by the dual edges of the bipartition edges is connected.

This condition is shown to be sufficient in [3] with the observation that the vertices of $H_{D}$ all have even degree (since any face of $G$ has an even number of incidences to bipartition edges). This implies that $H_{D}$ is Eulerian and that it is possible to construct $y$-monotone HH layouts with few bends in linear time.

The construction is roughly as follows: First, find a crossing-free Eulerian circuit $C_{H_{D}}$ of $H_{D}$ that separates the sets $V_{1}$ and $V_{2}$. Such a circuit $C_{H_{D}}$ exists since each crossing can be removed by swapping crossing pieces of the Eulerian circuit until all crossings are removed. Then dummy vertices $V^{\prime}$, that will become bends later, are introduced along the bipartition edges of $G$ to form $G^{\prime}$. Let $H_{1}^{\prime}$ be the induced subgraph 


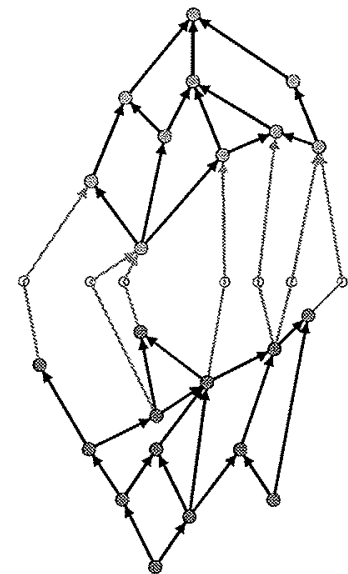

(a) Sample HH layout.

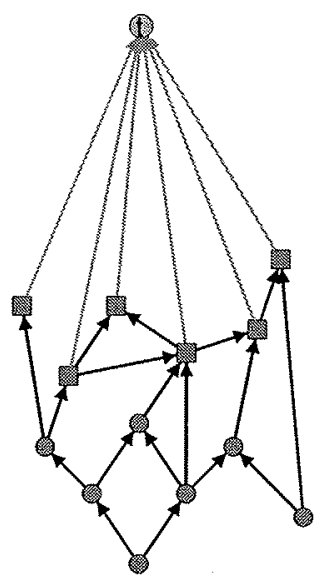

(b) Extended layout.

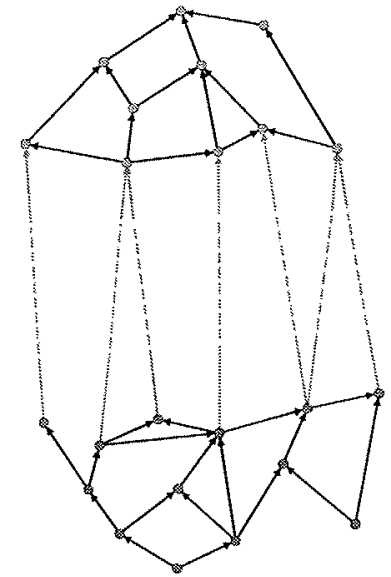

(c) Resulting embedding.

Fig. 4 HH layouts of $G$

of $G^{\prime}$ on $V_{1} \cup V^{\prime}$ along with these dummy vertices, and $H_{2}^{\prime}$ be the induced subgraph on $V_{2} \cup V^{\prime}$. Clearly, the union of $H_{1}^{\prime}$ and $H_{2}^{\prime}$ forms $G^{\prime}$. Since $C_{H_{D}}$ is crossing-free, there exists a planar drawing of $G^{\prime}$ such that $H_{1}^{\prime}$ and $H_{2}^{\prime}$ are separated by a simple closed curve.

This simple curve can be split arbitrarily such that the algorithm of Chrobak and Kant [6] can produce a straight-line drawing of $H_{1}^{\prime}$ and $H_{2}^{\prime}$ such that all the dummy vertices $V^{\prime}$ lie along a horizontal separation line $\ell$ in the order that the vertices of $V^{\prime}$ occur along $C_{H_{D}}$. Without loss of generality, $H_{2}^{\prime}$ can be placed above $\ell$ and $H_{1}^{\prime}$ can be flipped to lie below $\ell$, so that the final result is a straight-line planar drawing of $G^{\prime}$. This then is a straight-line drawing of $G$ with the exception of the bipartition edges that have exactly one bend each; see Fig. 4(a).

Next we show how to extend this result to obtain a drawing with no bends, while not introducing any crossings, after applying the above technique to the planar bipartition and obtaining the $\mathrm{HH}$ layout (which may have some bends).

Lemma 6 From each HH layout with some bends on the separation line $\ell$, we can derive a straight-line drawing with the two partitions separated by $\ell$.

Proof We begin by directing all the edges upward with respect to the basic $\mathrm{HH}$ layout $L$ in order to obtain an upward planar embedding $E$ of $G$. Any upward planar embedding $E$ can be realized as a straight-line upward drawing [9]. The resulting drawing, however, may not separate the two sets by a straight horizontal line. Below we show how to obtain the needed straight-line drawing such that the two sets are indeed separable by a line.

Let $\Gamma_{1}$ be the upward embedding of the graph $G_{1}$ with upper boundary $B_{1}$ of the vertices incident to the bipartition edges. We extend $\Gamma_{1}$ by adding a top vertex $t$ that we connect to all the boundary vertices with edges $(v, t)$ for each $v \in B_{1}$. We can apply the straight line drawing algorithm of Di Battista and Tamassia to the extended embedding and obtain an upward straight-line drawing. Since edges incident to $t$ proceed in a clockwise fashion in the same order that the vertices occur along the boundary of $B_{1}$, this implies that the path induced on $B_{1}$ is $x$-monotone; 
see Fig. 4(b). After removing vertex $t, B_{1}$ is once again the upper boundary. Similarly, we can extend the embedding $\Gamma_{2}$ of $G_{2}$ in order to obtain a drawing with $x$-monotone lower boundary $B_{2}$.

Next we stretch the two layouts in the $x$-direction so that the slopes of all edges are less than $30^{\circ}$. Stretching preserves both planarity and upwardness of the layouts. Finally, we place the two layouts of $\Gamma_{1}$ and $\Gamma_{2}$ (minus $t$ ) at a vertical distance larger than $\sqrt{3}$ times the maximum width of $\Gamma_{1}$ and $\Gamma_{2}$. We can safely insert the bipartition edges which connect the two boundaries $B_{1}$ and $B_{2}$. By the property of $30^{\circ}-60^{\circ}-90^{\circ}$ triangles, the slopes of all bipartition edges are larger than $60^{\circ}$. In particular, the slopes are larger than the maximum slope of $30^{\circ}$ of edges along the boundaries $B_{1}$ and $B_{2}$ so that no bipartition edge can cross a boundary edge. The two parts can now be separated by a horizontal line as desired; see Fig. 4(c).

Lemma 1 and the algorithm above yield the following lemma:

Lemma 7 Let $G$ be an n-vertex 2-colored planar graph with $r$ red vertices and $b$ blue vertices such that $n=r+b$ and the dual graph of the subgraph induced by the edges with endpoints of different colors is connected. Then there exists an $(r, b)-$ separable pointset onto which $G$ can be simultaneously embedded with any number of 2-colored paths with $r$ red vertices and $b$ blue vertices.

An outerplanar graph has a dual such that there is a vertex (corresponding to the outerface) that is adjacent to all other faces except for the faces where each adjacent face is one of these other faces. This implies that the dual of any induced subgraph must be connected. Hence, any 2-colored outerplanar graph fulfills the conditions of Lemma 7, which gives the following theorem:

Theorem 8 For every 2-colored $n$-vertex outerplanar $G$ there exists a 2-colored universal pointset $P$ of size $n$ onto which $G$ can be simultaneously embedded with any number of correspondingly 2-colored n-vertex paths.

\subsection{Paths on Three Colors}

We recall that there exist three paths which do not admit a simultaneous embedding with mapping [5], whereas, any number of paths can have a simultaneous embedding without mapping. Recall also that these two problems correspond to using $|V|$ and 1 colors, respectively. Restricting the pointsets of two colors to separate halfplanes suffices to allow any $(r, b)$-separable pointset to form a universal pointset for 2 -colored paths. We next show how to generalize this result to 3 -colored pointsets.

Theorem 9 There exists a 3-colored universal pointset $P$ of size $n$ onto which any number of correspondingly 3-colored $n$-vertex paths can be simultaneously embedded.

Proof Let $G$ be any 3-colored path with $r$ red vertices, $b$ blue vertices, and $g$ green vertices such that $r+b+g=n$. Using radial coordinates, we place $r$ red points at the 


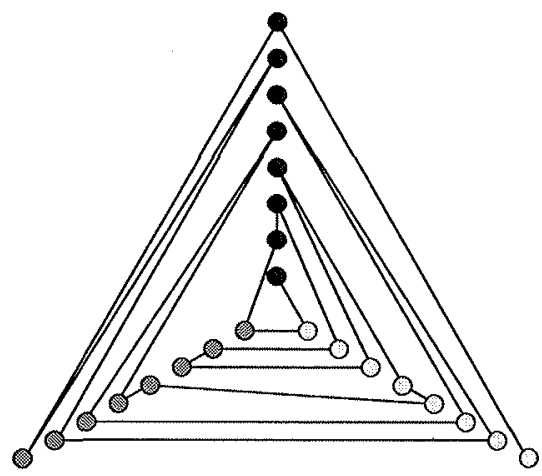

Fig. 5 Example embedding of a 3-colored path $G$ on a 3-colored universal pointset $P$

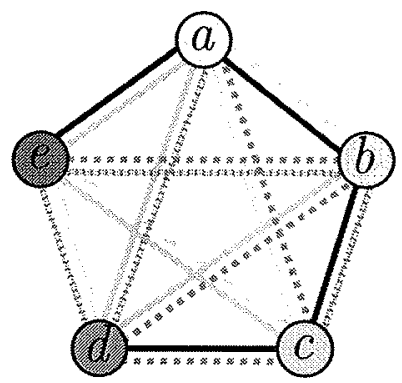

Fig. 6 Five 5-colored paths without a simultaneous embedding

coordinates $\left(\tau_{r}, 0\right)$ for $\tau_{r} \in\{1, \ldots, r\}, b$ blue points at the coordinates $\left(\tau_{b}, 2 \pi / 3\right)$ for $\tau_{b} \in\{1, \ldots, b\}$, and $g$ green points at the coordinates $\left(\tau_{g}, 4 \pi / 3\right)$ for $\tau_{g} \in\{1, \ldots, g\}$ to form pointset $P$; see Fig. 5. We next embed vertex $v_{j}$ of the path $G$, in order from $j=1, \ldots, n$, to the unused point $p_{j}$ of $P$ with the same color as $v_{j}$ that is the nearest to the origin. After embedding vertex $v_{i}$ along the path for some $i \in\{1, \ldots, n-1\}$, the convex hull of the points $\left\{p_{1}, \ldots, p_{i}\right\}$ forms triangle $T_{i}$. Given that for each remaining point $p \in P-\left\{p_{1}, \ldots, p_{i}\right\}$ and each corner $q$ of $T_{i}$, the line segment $\overline{p q}$ does not intersect $T_{i}$ except at $q$, the resulting drawing must be planar.

\subsection{Paths on Four and More Colors}

While universal $n$-vertex pointsets exist for paths with at most 3 colors, it is unknown whether one exists for 4-colored paths of arbitrary length. We next show there is no universal pointset for $k$-colored paths for any integer $k>4$.

Theorem 10 There does not exist a 5-colored universal pointset $P$ of size $n$ for any number of n-vertex correspondingly 5-colored paths.

Proof For our counterexample, we consider the following five 5-colored paths on 5 distinctly colored points in general position given in Fig. 6 whose union is $K_{5}$ where each edge in the $K_{5}$ belongs to exactly two paths:

(1) $a-c-d-b-e$ (thin red dashed edges),

(2) $a-d-e-b-c$ (thick light purple alternating dash and dot edges), 
(3) $b-a-c-e-d$ (thick green dotted edges),

(4) $b-d-a-e-c$ (thick yellow solid edges), and

(5) $e-a-b-c-d$ (thin blue solid edges).

Any straight-line drawing of $K_{5}$ on five distinct points has at least one crossing [20]. The crossing must be between two non-incident edges in the drawing of the $K_{5}$ union given that the points are in general position and no three points are co-linear so that no two incident edges can overlap. The paths above were chosen so that every pair of non-incident edges in the $K_{5}$ union belongs to the same path. Hence, that path must have a crossing between those two corresponding edges.

\section{Universal Pointsets for Trees}

We present universal pointsets of cardinality $n+\alpha$, where $\alpha \in\{0,1,3\}$ for three classes of $n$-vertex trees. The first pointset for 3-colored caterpillars has size $n$. The second pointset for 3-colored radius-2 stars has size $n+3$, in general, and size $n+1$ in a restricted case, so that not every point is used in every radius- 2 star. The last pointset for 2-colored spiders is easily seen to have size $n+2$. By using alternate embedding approaches for roots of different colors, two points can be eliminated to get an optimal pointset of size $n$.

Caterpillars, radius-2 stars, and degree- 3 spiders (with three legs) form the class of unlabeled level planar (ULP) trees [12], which has two known practical applications. First, the question posed in [5] of whether a path and a tree always have a simultaneous embedding with mapping remains open. Even if this cannot always be done, the question would become for which trees this is possible. The class of ULP trees partially addresses this more general question in that these trees are the only ones that can always be simultaneously embedded with any monotone path. Second, the ULP trees were used in showing that the set of minimum level non-planar (MLNP) patterns of Healy et al. [18] is incomplete [13].

\subsection{Caterpillars on Three Colors}

Recall that caterpillars are trees where the removal of all degree-1 vertices results in a path. Here we show that there exists a universal pointset for 3-colored caterpillars.

Theorem 11 There exists a 3-colored universal pointset $P$ of size $n$ onto which any number of n-vertex correspondingly 3-colored caterpillars can be simultaneously embedded.

Proof Let $T$ be any 3-colored caterpillar on colors $c_{1}, c_{2}$, and $c_{3}$ such that $\left|c_{1}\right|+$ $\left|c_{2}\right|+\left|c_{3}\right|=n$. Using radial coordinates, for $i \in\{1,2,3\}$, place $\left|c_{i}\right|$ points of color $c_{i}$ at the coordinates $\left(\tau_{i}+n, 2 \pi i / 3\right)$ for $\tau_{i} \in\left\{1, \ldots,\left|c_{i}\right|\right\}$.

Perturb the points of each color in a clockwise direction so that points of color $c_{i}$ lie along a common circular arc $A_{i}$ with no point perturbed by more than a sufficiently small distance $\varepsilon>0$ such that the following condition holds: For each point $p$ along 


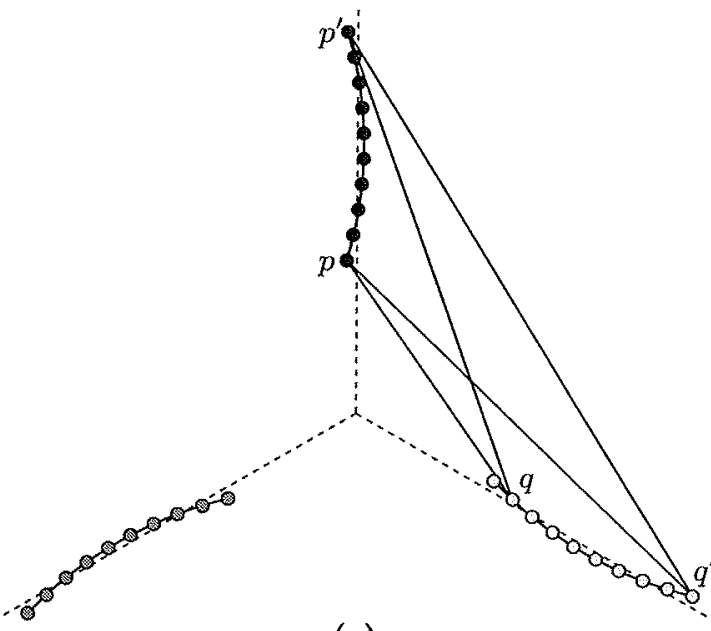

(a)

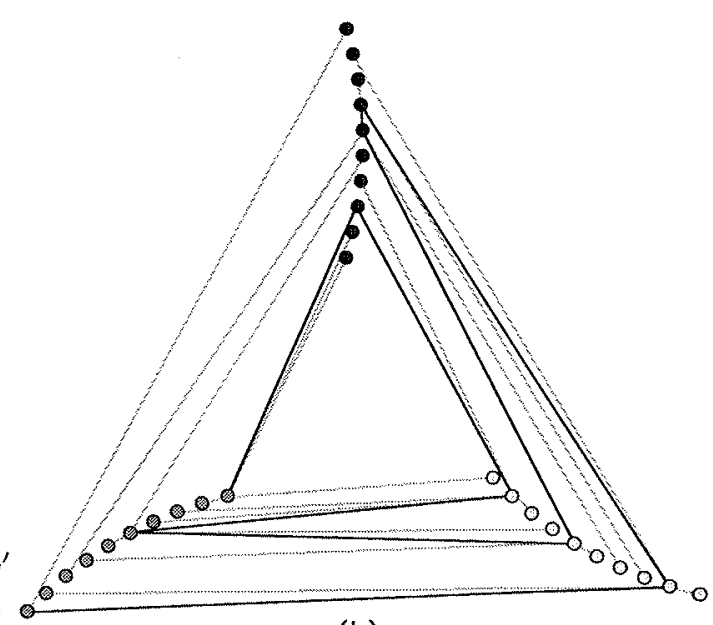

(b)

Fig. 7 The universal pointset $P$ for 3-colored caterpillars in (a) has the property that the line segment $\overline{p q}$ for any pair of points $p$ and $q$ does not intersect any of the circular arcs onto which the points lie. This allows for any caterpillar to be drawn on $P$ without crossings as in (b)

$\operatorname{arc} A_{i}$ and for each point $q$ along arc $A_{(i \bmod 3)+1}$, the line segment $\overline{p q}$ does not intersect either arc except at $p$ and $q$; see Fig. 7(a). Let $P$ denote this pointset.

Suppose that the spine $S$ (the path after all leaves are removed) of the caterpillar $T$ has $m$ vertices, $v_{1}, \ldots, v_{m}$, enumerated in order along $S$ from one endpoint $v_{1}$ to the other endpoint $v_{m}$. Let $i$ be an integer counter starting at 1 . For each $j=1, \ldots, m$, we first embed $v_{j}$ onto the unused point $p_{i}$ of $P$ that is nearest to the origin with the same color as $v_{j}$ and increment $i$. Before embedding the next spine vertex $v_{j+1}$, we next embed any leaf vertex $u_{j, k}$ of $v_{j}$ for $k \in\left\{1, \ldots\right.$, degree $\left.\left(v_{j}\right)-2\right\}$, onto the next available point $p_{i}$ in $P$ that is closest to the origin with the same color as $u_{j, k}$, taking care to increment $i$ for each vertex embedded; see Fig. 7(b). The convex hull of the points $\left\{p_{1}, \ldots, p_{i}\right\}$ forms a triangle $T_{i}$ for each $i \in\{1, \ldots, n\}$. Given that for each remaining point $p \in P-\left\{p_{1}, \ldots, p_{i}\right\}$ and each corner $q$ of $T_{i}$, the line segment $\overline{p q}$ does not intersect $T_{i}$ except at $q$, the resulting drawing must be planar.

\subsection{Radius-2 Stars on Three Colors}

A radius-2 star is a tree consisting of any number of paths, or legs, of length at most 2 sharing a common endpoint $r$, the root. We show how to simultaneously embed a radius- 2 star on $n+3$ points. If we constrain each color class to have the same size, $n / 3$, and do not permit legs of length 1 , we reduce the pointset to $n+1$ points.

Theorem 12 There exists a 3-colored universal pointset $P$ of size $n+3$ onto which any number of n-vertex correspondingly 3-colored radius-2 stars can be simultaneously embedded.

Proof Let $T$ be any 3-colored radius-2 star on colors $c_{1}, c_{2}$, and $c_{3}$, where $\left|c_{1}\right|+$ $\left|c_{2}\right|+\left|c_{3}\right|=n$. Place one point $p_{i}$ of each color $c_{i}$ at $(i-2,0)$ so that $p_{2}$ lies at the origin $O, p_{1}$ lies one unit left of $O$, and $p_{3}$ lies one unit to the right of $O$. Let $A$ 


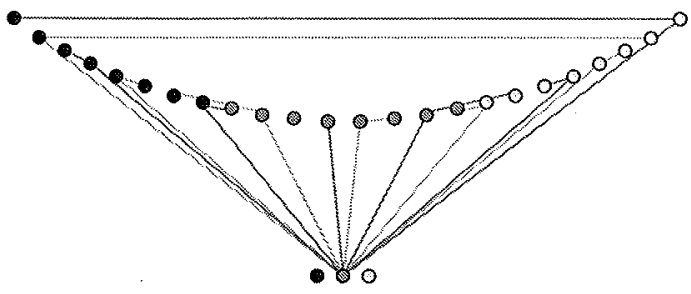

(a)

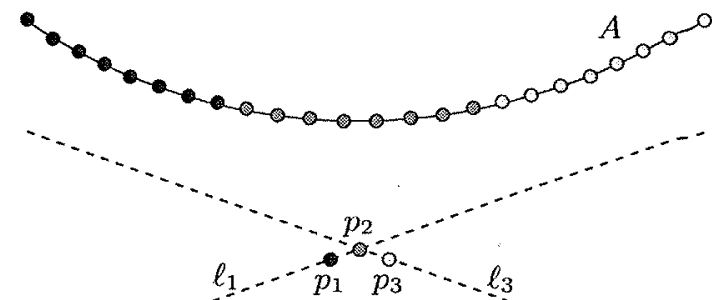

(b)

Fig. 8 The universal pointset for 3-colored radius-2 stars (a) has the property shown in (b) that all the points along the arc $A$ are contained in the half-planes given by the lines, $\ell_{1}$ and $\ell_{3}$

be a concave circular arc centered above $O$ that is visible in its entirety from each point $p_{i}$. Place $\left|c_{1}\right|$ points of color $c_{1}$ along the leftmost part of the arc $A$, followed by $\left|c_{2}\right|$ points of color $c_{2}$ along the central part of $A$, and then $\left|c_{3}\right|$ points of color $c_{3}$ along the rightmost part of $A$; see Fig. 8(b).

We contend that these $n+3$ points comprise a universal pointset for $T$. Place the root $r$ of $T$ (the vertex of maximum degree) on the corresponding point $p_{i}$ of its color $c_{i}$, and call this point $X$. Each leg of $T$ will be drawn from $X$ to a point $Y$ along the arc $A$ with the line segment $\overline{X Y}$, and if the leg has length 2, from $Y$ to $Z$ along $A$ with the additional line segment $\overline{Y Z}$. We need to ensure that the appropriate points $Y$ and $Z$ are selected for each leg of length 2 in order to avoid crossings.

We denote the legs of $T$ of length 1 as $r-s$ and the legs of length 2 as $r-s-t$ where vertices $s$ and $t$ have colors $c_{j}$ and $c_{k}$, respectively. We start by drawing each leg $r-s$ of length 1 where we pick the free point nearest to $X$ of the color $c_{j}$ to be the point $Y$. We next draw the legs $r-s-t$ where $c_{j}$ and $c_{k}$ differ. If neither $c_{j}$ nor $c_{k}$ is $c_{2}$, we pick the free points furthest from $X$ of colors $c_{j}$ and $c_{k}$, respectively, to be the points $Y$ and $Z$. In this case, the triangle $\triangle X Y Z$ contains all remaining points.

Otherwise, either $c_{j}$ or $c_{k}$ is $c_{2}$. We pick the free points that consist of the furthermost free point of color $c_{2}$ and the nearest free point of the other color $c_{1}$ or $c_{3}$, the colors $c_{j}$ and $c_{k}$, respectively, to be the points $Y$ and $Z$. In this case, the triangle $\triangle X Y Z$ only contains previously used points of colors $c_{j}$ and $c_{k}$. Finally, we draw the legs $r-s-t$ where $s$ and $t$ share the same color $c_{j}$. We pick the remaining two points nearest to $X$ of color $c_{j}$ to be the points $Y$ and $Z$. Given that any points between $Y$ and $Z$ along $A$ must also be of color $c_{j}$, the triangle $\triangle X Y Z$ contains no unused points.

For each leg, all remaining free points either lie inside or outside of the respective triangle $\triangle X Y Z$. Hence, the line segment $\overline{Y Z}$ cannot cross any previous line segment; see Fig. 8(a). The final result is a plane drawing of $T$.

We should note that for some colorings of a radius- 2 star, we can reduce the size of the pointset by always using the points $p_{1}$ and $p_{3}$. For instance, if the root has color $c_{2}$ so that it uses $p_{2}$, then any leg with all three colors can be drawn using $p_{1}$ and $p_{3}$ if they are placed below $p_{2}$. Alternately, if there is a leg such that the two non-root vertices have the color $c_{1}$ (or $c_{3}$ ), then the leg can be drawn using $p_{1}$ (or $p_{3}$ ) and the uppermost point of color $c_{1}$ (or $c_{3}$ ). 


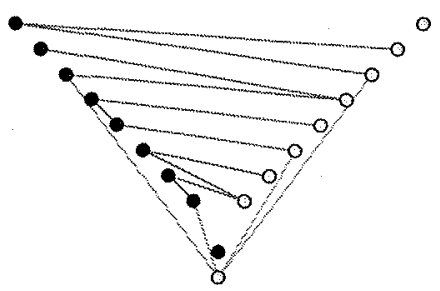

(a)

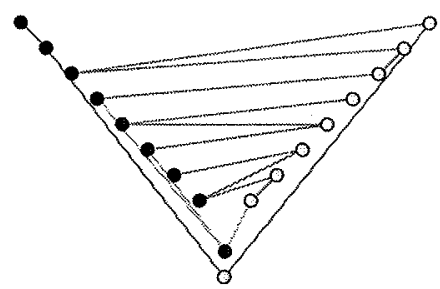

(b)

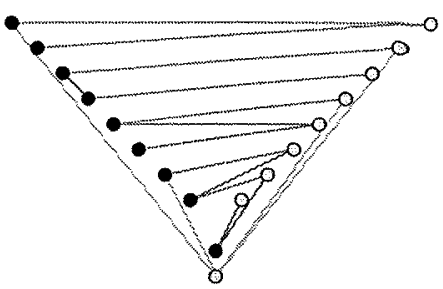

(c)

Fig. 9 The universal pointset in (a) for 2-colored spiders starts with size $n+2$. One point of each color can be removed to allow for a universal pointset of size $n$ as in (b) and (c)

\subsection{Spiders on Two Colors}

A spider is a tree that is obtained by subdividing a star so that its "legs" have arbitrary length. We present a universal pointset of size $n$ for $n$-vertex 2 -colored spiders. We first show how to embed any spider on a larger pointset of size $n+2$ and then show how to eliminate the two extra vertices, one of each color.

Lemma 13 There exists a 2-colored universal pointset $P$ of size $n+2$ onto which any number of n-vertex correspondingly 2-colored spiders can be simultaneously embedded.

Proof Let $T$ be any 2-colored spider on colors $c_{1}$ and $c_{2}$, where $\left|c_{1}\right|+\left|c_{2}\right|=n$. Let $\ell_{1}$ and $\ell_{2}$ be two rays with a common endpoint $O$ at the origin meeting at a $90^{\circ}$ angle; see Fig. 9(a). Start by placing $\left|c_{i}\right|$ points along $\ell_{i}$ so that the first point of each color $\ell_{i}$ is at the nearest integer grid position (i.e., $(1,1)$ and $(-1,1)$ ). The remaining points are consecutive integer grid points along the lines $\ell_{i}$. Next we place one point $p_{i}$ of each color $c_{i}$ directly below the origin at positions $(0,-i)$, i.e., $(0,-1)$ and $(0,-2)$.

We show that this pointset is universal for $T$. Place the root vertex $r$ on the point of the correct color below the origin. For each leg (a 2-colored path rooted at $r$ ), place its vertices at the correctly colored free point nearest to the origin $O$. The first path is drawn without crossings, as consecutive points along the path are either of the same color, in which case there clearly are no crossings, or are of different color, which leads to the path zig-zagging between points on $\ell_{1}$ and $\ell_{2}$. In the latter case, there are no crossings as the path always takes vertices farther away from the origin.

For the $k$ th leg in the spider, the previous paths have taken a set of consecutive points of each color, defining a triangle $\Delta_{k-1}$, determined by $r$ and the last two taken points, farthest from the origin along $l_{1}$ and $l_{2}$. The $k$ th leg is embedded as before, by placing its vertices at the correctly colored free point nearest to the origin $O$. As before, the edges of the $k$ th leg do not cross each other as the path zig-zags farther and farther away from $O$. The path does not enter the triangle $\Delta_{k-1}$ as all but the first edge of the $k$ th leg are above $\Delta_{k-1}$, and the first edge stays clear of $\Delta_{k-1}$ as it goes from $r$ to a point either strictly to the left or to the right of $\Delta_{k-1}$.

Next, we consider the two colorings of the root of the spider to show that we can always use the other point below the origin, thereby reducing the pointset to size $n$. 
Theorem 14 There exists a 2-colored universal pointset $P$ of size $n$ onto which any number of $n$-vertex correspondingly 2-colored spiders can be simultaneously embedded.

Proof We start with the pointset given in Lemma 13 and instead of placing $\left|c_{i}\right|$ points along $\ell_{i}$, we only place $\left|c_{i}\right|-1$ points. The resulting set of points is of the desired size $n$ and, as we show below, is universal for 2-colored spiders.

First, we consider spiders where the root, $r$, has color $c_{1}$. As before, we place $r$ on the point $p_{1}$ at $(0,-1)$. We need to show that it always possible to use the point $p_{2}$ at $(0,-2)$ of color $c_{2}$ for some leg of the spider. We use the next available correctly colored point that is nearest to $O$ with one notable exception: We ignore the point $p_{2}$ below $r$ until we encounter the last vertex of color $c_{2}$ in drawing $T$ in which case we use $p_{2}$; see Fig. 9(b). This prevents the leg from self-intersecting when drawn-any edges incident to $p_{2}$ must lie along the convex hull of the points used to draw the leg.

Next, we consider spiders where the root is colored $c_{2}$. We place $r$ on the point $p_{2}$ at $(0,-2)$. We attempt to follow the previous strategy from Lemma 13 . We draw each leg as before starting with the next available point that is nearest to $O$ of the correct color, but this time we use the point $p_{1}$ as the first free point for the color $c_{1}$. However, this may not always work if the vertices of the first leg, $r-a-b-c-\cdots$, strictly alternate between the colors $c_{1}$ and $c_{2}$ such that $a$ and $c$ have color $c_{1}$ and $b$ has color $c_{2}$. In this case, edge $(r, a)$ crosses edge $(b, c)$.

To avoid this, we revise our strategy by reversing the order in which we draw each leg. We start with the next available correctly colored point that is the furthest from $O$, instead of the one nearest to $O$; see Fig. 9(c). Point $p_{1}$ is the point of color $c_{1}$ nearest to $O$, and hence, $p_{1}$ will be chosen as the last point for color $c_{1}$ instead of being chosen first.

The $(k+1)$ th leg is fully contained inside the triangle $\Delta_{k}$ formed by $r$ and the two taken points along $\ell_{1}$ and $\ell_{2}$, nearest to the origin $O$, along the $k$ th leg. These triangles nest as before in Lemma 13, so no crossings are introduced between legs. Given that we avoid crossings while drawing each leg, a plane drawing is the result. $\square$

\section{Universal Pointsets for Some Outerplanar Graphs}

We extend the previous pointsets to accommodate any outerplanar graph which has as a spanning tree one of the trees from the previous section (caterpillars, radius-2 stars, and degree-3 spiders).

\section{1 $K_{3}$-Caterpillars on Three Colors}

A $K_{3}$-caterpillar is an extension of a caterpillar such that for each spine edge $(u, v)$ of the caterpillar, two additional edges $(u, w)$ and $(w, v)$ and a vertex $w$ of degree 2 can be added to the graph so that instead of the spine edge $(u, v)$ there is a $K_{3}$ on the vertices $\{u, v, w\}$ in its place. The resulting graph is no longer a tree given the introduction of cycles, but is an outerplanar graph with similar structure. We take advantage of this structural similarity to show that the universal pointset for 3-colored caterpillars is also a universal pointset for 3-colored $K_{3}$-caterpillars. 


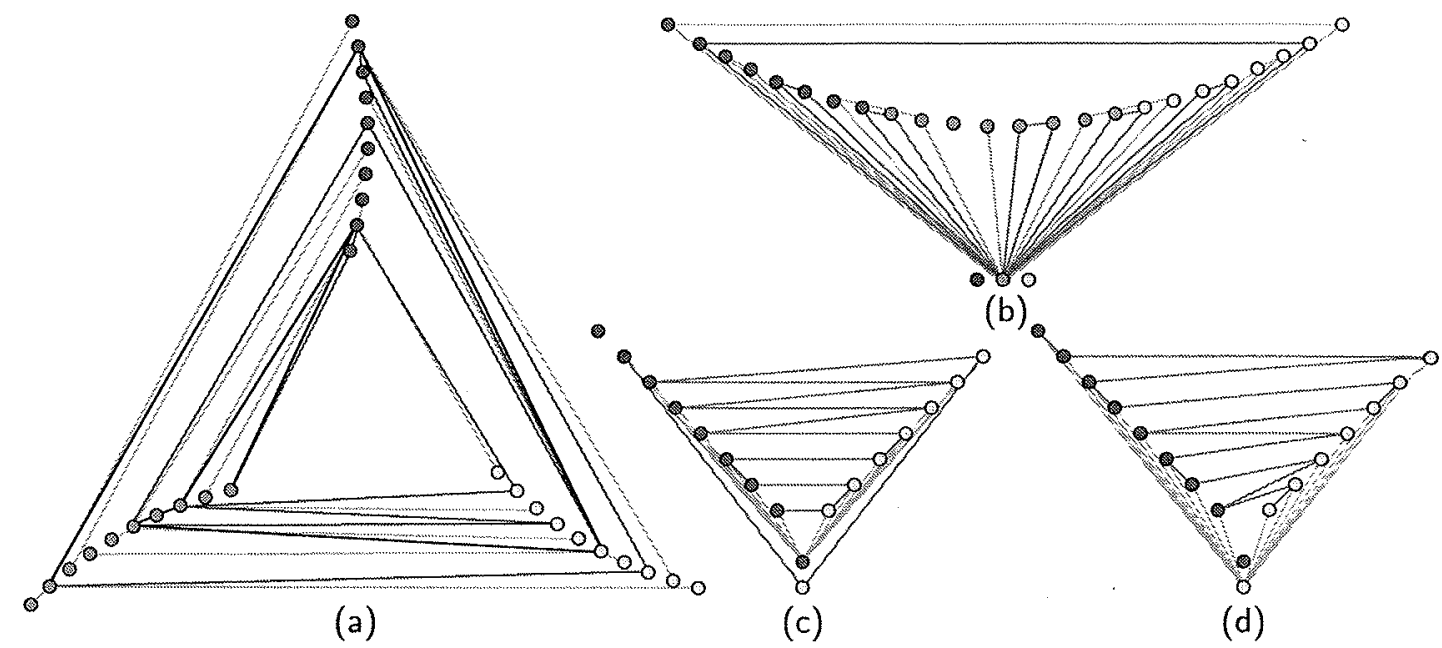

Fig. 10 Universal pointsets of size $n$ for 3-colored $K_{3}$-caterpillar in (a), of size $n+3$ for 3-colored $K_{3}$-stars in (b), and of size $n+1$ for 2-colored fans in (c) and (d)

Theorem 15 There exists a 3-colored universal pointset $P$ of size $n$ onto which any number of $n$-vertex correspondingly 3 -colored $K_{3}$-caterpillars can be simultaneously embedded.

Proof We use the same pointset as in Theorem 11. We proceed to draw the $K_{3}$-caterpillar as before where we draw legs before cut edges that are incident to the same cut vertex. However, we need to be careful when drawing the additional edges of each $K_{3}$ so as to avoid crossings. In drawing each $K_{3}$ that consists of the cut-edge $(u, v)$ and the edges $(u, w)$, and $(w, v)$ (vertex $w$ is of degree 2$)$, we first draw the edges in the order $(u, w),(w, v)$, and $(u, v)$, before drawing any other edges incident to $u$, using the first available point of the correct color. This ensures that the edge $(u, v)$ does not cross any other edge since the point $v$ is still visible from $u$; see Fig. 10(a). Hence, a plane drawing results.

\section{$4.2 K_{3}$-Stars on Three Colors}

A $K_{3}$-star is a star with root $r$ such that for each pair of leaves $s$ and $t$ of degree-1, an edge $(s, t)$ can be added to the graph so that the vertices $s$ and $t$ will have degree-2 in the final graph. In Theorem 12, each leg of a radius-2 star consists of two of the three edges of a triangle formed by the points $r, s$, and $t$. At any one point, the leg $r-t-s$ could be substituted for the leg $r-s-t$ so that a $K_{3}$ could be drawn on the points $r$, $s$, and $t$ without introducing crossings; see Fig. 10(b). This gives the following two theorems:

Theorem 16 There exists a 3-colored universal pointset $P$ of size $n+3$ onto which any number of $n$-vertex correspondingly 3 -colored $K_{3}$-stars can be simultaneously embedded.

Theorem 17 There exists a universal pointset $P$ of size $n+1$ onto which any number of $n$-vertex 3-colored $K_{3}$-stars can be simultaneously embedded provided that each color class has the size $n / 3$ and no vertices have degree 1 . 


\subsection{Fans on Two Colors}

While a spider is formed by connecting an arbitrary number of paths to a single root vertex, a fan is formed by connecting all vertices of a single path to the root vertex. Clearly, every spanning tree of a fan is a spider, but not every graph with a spider as a spanning tree is a fan. Analogous statements can be made for caterpillars and $K_{3}$-caterpillars and for radius- 2 stars and $K_{3}$-stars. However, unlike the previous results of this section, the size of the universal pointset for the graph is not the same as the size of the universal pointset that can be obtained for one of its spanning trees.

Theorem 18 There exists a 2-colored universal pointset $P$ of size $n+1$ onto which any number of n-vertex correspondingly 2-colored fans can be simultaneously embedded.

Proof We use the same pointset used in Theorem 14 except that we need an additional point of color $c_{1}$ along $\ell_{1}$. Let $s$ and $t$ denote the vertices adjacent to $r$ along the outerface of the fan, and let $s \leadsto t$ denote the maximal path along the outerface that does not include $r$. We proceed to draw $s \rightsquigarrow t$ using a strategy based upon Theorem 14. We pick the nearest correctly colored free point first with two exceptions: (1) we ignore $p_{1}$ if the root has color $c_{2}$ in order to avoid a potential crossing (if the coloring of the outerface is strictly alternating) and (2) we only use $p_{2}$ for the last vertex of color $c_{2}$ if the root has color $c_{1}$. As a result, $p_{2}$ is always used, but either $p_{1}$ or the point furthest from $O$ of color $c_{1}$ is not used. In both cases, the path $s \rightsquigarrow t$ is drawn without a self-intersection, and all points along the path are visible from point $r$. Hence, an edge can then be added from $r$ to each of the other $n-1$ points in the pointset onto which $s \rightsquigarrow t$ was drawn. This clearly yields a plane drawing.

\section{More Graphs Without Simultaneous Embeddings}

We next consider graphs without simultaneous embeddings for $2 \leq k \leq 9$ colors.

\subsection{Two Colors}

Fowler et al. [14] presented a set of 16 pairs of planar graphs that cannot always be simultaneously embedded whose unions are either homeomorphic to $K_{5}$ or to $K_{3,3}$. Most of these pairs require that the vertices be distinctly colored. However, the 5-vertex pair given in Fig. 11(a) can be 2-colored and still not always have a simultaneous embedding as we show next.

Theorem 19 There exist a 2-colored planar graph and a pseudo-forest that cannot be simultaneously embedded.

Proof Let $G_{1}$ be the planar graph consisting of a $K_{5}$ minus an edge $e$ in which the endpoints of the missing edge are red and the remaining three vertices are blue. Let $G_{2}$ be the pseudo-forest consisting of a $K_{3}$ on three blue vertices and an non-incident 


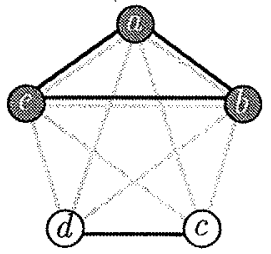

(a)

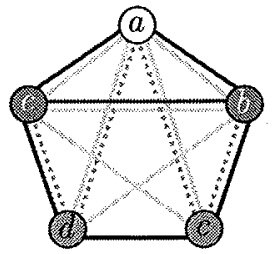

(b)

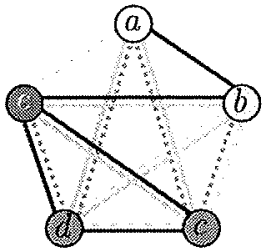

(c)

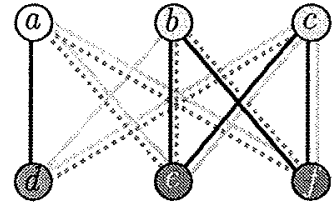

(d)

Fig. 11 A planar graph and a pseudo-forest on 2 colors in (a), three outerplanar graphs on 3 colors in (b), four pseudo-forests on 4 colors in (c), and three pseudo-forests on 5 colors in (d) without simultaneous embeddings

edge on two red vertices; see Fig. 11(a). In both $G_{1}$ and $G_{2}$, the vertices with the same color have the same adjacencies. Hence, there is only one non-isomorphic mapping of the vertices onto the three blue points and the two red points. Each pair of nonincident edges in the non-planar $K_{5}$ union is present in one of the graphs. In any straight-line embedding of the vertices of these graphs in the plane where incident edges do not overlap, at least one of the graphs must have a crossing, which prevents a simultaneous embedding.

\subsection{Three Colors}

We next show that there exist outerplanar triples on 3 colors that cannot be simultaneously embedded.

Theorem 20 There exist three 3-colored outerplanar graphs that cannot be simultaneously embedded.

Proof Consider the following three 3-colored outerplanar graphs (the third is a path) with 5 vertices embedded onto the 5 points as given in Fig. 11(b) whose union forms a $K_{5}$ :

(1) $a-b-c-d-e-a$ and $b-e$ (dark blue edges),

(2) $a-c-e-b-d-a$ and $b-a-e$ (light yellow edges),

(3) $b-c-a-d-e$ (dashed red edges),

where $a$ is colored yellow, $b$ and $e$ are colored blue, and $c$ and $d$ are colored red.

For each graph, vertices with the same color have the same adjacencies. Hence, there is only one non-isomorphic mapping of the vertices onto one yellow point, two blue points and two red points. Each pair of non-incident edges in the non-planar union is in one of the three graphs, forcing at least one of the graphs to have a crossing in any given straight-line drawing where two incident edges do not overlap.

\subsection{Four and Five Colors}

We next turn our attention to pseudo-forests, a proper subclass of outerplanar graphs.

Theorem 21 There exist four 4-colored pseudo-forests and three 5-colored pseudoforests that cannot be simultaneously embedded. 
Proof Consider the following four 4-colored pseudo-forests shown in Fig. 11(c) whose union is $K_{5}$ :

(1) $a-b-e-c-d-e$ (dark blue edges),

(2) $a-c-e-b-d-a$ (light yellow edges),

(3) $b-c-a-d-e$ (dashed red edges),

(4) $b-c-d-b$ and $a-e$ (dashes alternating with 3 dots green edges),

where only $c$ and $d$ have the same color and the following three 5-colored pseudoforests shown in Fig. 11(d) whose union is $K_{3,3}$ :

(1) $a-d$ and $b-f-c-e-b$ (dark blue edges),

(2) $a-e-c-f-a$ and $c-d-b$ (light yellow edges),

(3) $a-e-b-f-a$ and $c-d$ (dashed red edges),

where only $e$ and $f$ share a color. For each graph, vertices with the same color have the same adjacencies, so there is only one non-isomorphic mapping of the vertices onto points of the same color. Each pair of non-incident edges in the non-planar union is in one of the graphs, forcing at least one graph to have a crossing.

\subsection{Six and Nine Colors}

A pair of outerplanar graphs on 6 vertices and a triple of paths on 9 vertices that do not always have a simultaneous embedding were given in [5], which in the context of colored simultaneous embeddings implies our final two theorems.

Theorem 22 There exist two 6-colored biconnected outerplanar graphs that cannot be simultaneously embedded.

Proof Consider the following two 6-colored outerplanar graphs shown in Fig. 12(a):

(1) $a-d-b-f-c-e-a$ and $b-e$ (dark blue edges), and

(2) $a-e-c-d-b-f-a$ and $b-e$ (light yellow edges).

For each edge of the $K_{3,3}$ union that is not in the intersection, all of its nonadjacent edges belong to the same graph. Since the vertices have distinct colors and $K_{3,3}$ is non-planar [20], this forces a crossing in one of the two graphs.

Theorem 23 There exist three 6-colored cycles, four 6-colored paths and three 9-colored paths that cannot be simultaneously embedded.

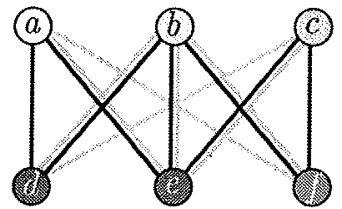

(a)

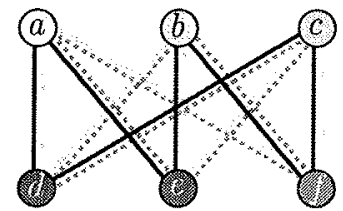

(b)

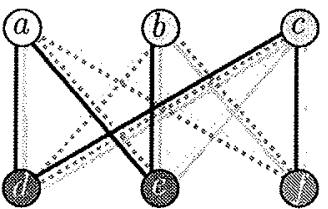

(c)

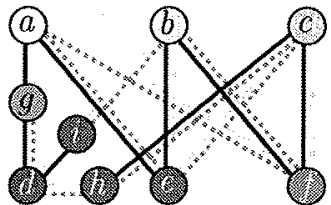

(d)

Fig. 12 Two biconnected outerplanar graphs on 6 colors in (a), three cycles and four paths on 6 colors in (b), (c), and three paths on 9 colors in (d) without a simultaneous embedding 
Proof Consider the following three 6-colored cycles shown in Fig. 12(b):

(1) $e-a-d-c-f-b-e$ (blue solid edges),

(2) $e-a-f-b-d-c-e$ (red dashed edges), and

(3) $a-f-c-e-b-d-a$ (green dotted edges);

and the following four 6-colored paths shown in Fig. 12(c):

(1) $e-a-d-c-f-b$ (blue solid edges),

(2) $e-a-f-b-d-c$ (red dashed edges),

(3) $a-f-c-e-b-d$ (green dotted edges), and

(4) $a-d-c-e-b-f$ (yellow solid edges);

and the following set of 9-colored paths shown in Fig. 12(d):

(1) $h-c-f-b-e-a-g-d-i$ (blue solid edges),

(2) $g-d-h-c-e-a-f-b-i$ (red dashed edges), and

(3) $g-a-f-c-e-b-i-d-h$ (green dotted edges).

All three sets of graphs have non-planar unions that form $K_{3,3}$ (or a subdivision of $\left.K_{3,3}\right)$. For both of the three cycles and four paths, every one of the 18 pairs of nonadjacent edges in the $K_{3,3}$ (subdivision) belong to a common cycle or path. Thus, at least one of the cycles or paths must be self-intersecting.

\section{Conclusions and Open Problems}

We introduced the problem of colored simultaneous embedding and the related problem of small sets supporting CSE and universal CSE pointsets.

We described how a given 2-colored tree or outerplanar graph can be embedded onto a set of 2-colored points in general position separated by a straight-line giving a universal pointset for 2-colored paths. It remains open, though, as to whether there exists a universal $(r, b)$-pointset for all 2-colored trees. We also presented a universal pointset for 3-colored paths, and showed none exist for 5-colored paths, leaving the question of whether there exists a universal pointset for 4-colored paths open.

We provided universal or near-universal pointsets for caterpillars and radius- 2 stars on 3 colors and degree- 3 spiders on 2 colors, which comprise the three classes of ULP trees [12].

In the context of CSE, previous examples of graphs without simultaneous embeddings, such as the 16 pairs given in [14], required that each vertex be distinctly colored. We presented several examples where this was not the case. We accomplished this by ensuring that vertices with the same color had the same adjacencies.

Table 1 summarizes the current status of colored simultaneous embedding. A " $\checkmark$ " indicates that it is always possible to simultaneously embed the type of graphs, a " $X$ " indicates that it is not always possible, and a "?" indicates an open problem. New results are in bold with asterisks.

Acknowledgements We would like to thank the anonymous reviewers for their insightful suggestions for improvement. Their comments led to simpler algorithms and easier arguments. 
Table $1 k$-colored simultaneous embeddings on $n$ points: new results and open problems

\begin{tabular}{|c|c|c|c|c|c|c|c|c|}
\hline & \multicolumn{8}{|l|}{$k$} \\
\hline & 1 & 2 & 3 & 4 & 5 & 6 & 9 & $n$ \\
\hline Three paths $P_{1} \ldots P_{3}$ & $\checkmark$ & $\checkmark$ & $\checkmark$ & $?$ & $?$ & $?$ & $\mathbf{x}[5]$ & $\mathbf{x}$ \\
\hline Four paths $P_{1} \ldots P_{4}$ & $\checkmark$ & $\checkmark$ & $\checkmark$ & $?$ & $?$ & $\mathrm{x}$ & $\mathbf{x}$ & $\mathbf{x}$ \\
\hline${ }^{* * *}$ Three cycles $C_{1} \ldots C_{3}$ & $\checkmark$ & $\checkmark$ & $\checkmark$ & $?$ & $?$ & $\mathrm{x}$ & $x$ & $\mathbf{x}$ \\
\hline Two outerplanar graphs $O_{1}, O_{2}[5,15]$ & $\checkmark$ & $?$ & $?$ & $?$ & $?$ & $\mathrm{x}$ & $x$ & $\mathbf{x}$ \\
\hline Three outerplanar graphs $O_{1} \ldots O_{3}$ & $\checkmark$ & $?$ & $\mathbf{x}$ & $\mathbf{x}$ & $\mathbf{x}$ & $\mathrm{x}$ & $\mathrm{x}$ & $\mathbf{x}$ \\
\hline${ }^{* * *}$ Three pseudo-forests $F_{1} \ldots F_{3}$ & $\checkmark$ & $?$ & $?$ & $?$ & $\mathbf{x}$ & $\mathbf{x}$ & $x$ & $\mathbf{x}$ \\
\hline *** Four pseudo-forests $F_{1} \ldots F_{4}$ & $\checkmark$ & $?$ & $?$ & $\mathbf{x}$ & $x$ & $\mathbf{x}$ & $\mathbf{x}$ & $\mathbf{x}$ \\
\hline${ }^{* * *}$ Any number of paths & $\checkmark$ & $\checkmark$ & $\checkmark$ & $?$ & $\mathbf{x}$ & $x$ & $\mathbf{x}$ & $\mathbf{x}$ \\
\hline${ }^{* * *}$ Any number of caterpillars & $\checkmark$ & $\checkmark$ & $\checkmark$ & $?$ & $\mathbf{x}$ & $\mathbf{x}$ & $\mathbf{x}$ & $\mathbf{x}$ \\
\hline Two trees $T_{1}, T_{2}[19]$ & $\checkmark$ & $?$ & $?$ & $?$ & $?$ & $?$ & $?$ & $x$ \\
\hline *** Tree $T$ and path $P$ & $\checkmark$ & $\checkmark$ & $?$ & $?$ & $?$ & $?$ & $?$ & $?$ \\
\hline${ }^{* * *}$ Outerplanar graph $G$ and path $P$ & $\checkmark$ & $\checkmark$ & $?$ & $?$ & $?$ & $?$ & $?$ & $?$ \\
\hline Planar graph $G$ and path $P[5,11]$ & $\checkmark$ & $?$ & $?$ & $?$ & $?$ & $?$ & $\mathbf{x}$ & $x$ \\
\hline${ }^{* * *}$ Planar graph $G$ and pseudo-forest $F$ & $?$ & $\mathbf{x}$ & $\mathbf{x}$ & $\mathbf{x}$ & $\mathbf{x}$ & $\mathbf{x}$ & $\mathbf{x}$ & $\mathbf{x}$ \\
\hline
\end{tabular}

\section{References}

1. Abellanas, M., García, J., Hernández, G., Noy, M., Ramos, P.: Bipartite embeddings of trees in the plane. Discrete Appl. Math. 93(2-3), 141-148 (1999)

2. Biedl, T.C.: Drawing planar partitions I: LL-drawings and LH-drawings. In: Proceedings of the Fourteenth Annual Symposium on Computational Geometry, SoCG 1998, pp. 287-296. ACM Press, New York (1998)

3. Biedl, T.C., Kaufmann, M., Mutzel, P.: Drawing planar partitions. II. HH-drawings. In: GraphTheoretic Concepts in Computer Science, Smolenice Castle, 1998. LNCS, vol. 1517, pp. 124-136. Springer, Berlin (1998)

4. Bose, P.: On embedding an outer-planar graph in a point set. Comput. Geom.: Theory Appl. 23(3), 303-312 (2002)

5. Brass, P., Cenek, E., Duncan, C.A., Efrat, A., Erten, C., Ismailescu, D., Kobourov, S.G., Lubiw, A., Mitchell, J.S.B.: On simultaneous graph embedding. Comput. Geom.: Theory Appl. 36(2), 117-130 (2007)

6. Chrobak, M., Kant, G.: Convex grid drawings of 3-connected planar graphs. Int. J. Comput. Geom. Appl. 7(3), 211-223 (1997)

7. de Fraysseix, H., Pach, J., Pollack, R.: Small sets supporting Fary embeddings of planar graphs. In: Proceedings of the 20th Annual ACM Symposium on Theory of Computing, STOC 1988, pp. 426433 (1988)

8. de Fraysseix, H., Pach, J., Pollack, R.: How to draw a planar graph on a grid. Combinatorica 10(1), 41-51 (1990)

9. Di Battista, G., Tamassia, R.: Algorithms for plane representation of acyclic digraphs. Theor. Comput. Sci. 61(23), 175-198 (1988)

10. Dux, B., Iyer, A., Debray, S., Forrester, D., Kobourov, S.G.: Visualizing the behavior of dynamically modifiable code. In: 13th Workshop on Program Comprehension, pp. 337-340 (2005)

11. Erten, C., Kobourov, S.G.: Simultaneous embedding of planar graphs with few bends. J. Graph Algorithms Appl. 9(3), 347-364 (2005)

12. Estrella-Balderrama, A., Fowler, J.J., Kobourov, S.G.: Characterization of unlabeled level planar trees. Comput. Geom.: Theory Appl. 42(7), 704-721 (2009)

13. Fowler, J.J., Kobourov, S.G.: Minimum level nonplanar patterns for trees. In: 15th Symposium on Graph Drawing, GD 2007. LNCS, vol. 4875, pp. 69-75. Springer, Berlin (2008) 
14. Fowler, J.J., Jünger, M., Kobourov, S.G., Schulz, M.: Characterizations of restricted pairs of planar graphs allowing simultaneous embedding with fixed edges. In: 34 th International Workshop on GraphTheoretic Concepts in Computer Science, WG 2008. LNCS, vol. 5344, pp. 146-158. Springer, Berlin (2008)

15. Frati, F.: Embedding graphs simultaneously with fixed edges. In: 14th Symposium on Graph Drawing, GD 2006. LNCS, vol. 4372, pp. 108-113. Springer, Berlin (2007)

16. Frati, F., Kaufmann, M., Kobourov, S.G.: Constrained simultaneous and near-simultaneous embeddings. In: 15th Symposium on Graph Drawing, GD 2007. LNCS, vol. 4875, pp. 268-279. Springer, Berlin (2008)

17. Gritzmann, P., Mohar, B., Pach, J., Pollack, R.: Embedding a planar triangulation with vertices at specified points. Am. Math. Mon. 98, 165-166 (1991)

18. Healy, P., Kuusik, A., Leipert, S.: Characterization of level non-planar graphs by minimal patterns. In: 6th Computing and Combinatorics Conference, COCOON 2000, pp. 74-84 (2000)

19. Kaufmann, M., Vrto, I., Geyer, M.: Two trees which are self-intersecting when drawn simultaneously. In: 13th Symposium on Graph Drawing, GD 2005. LNCS, vol. 3843, pp. 201-210. Springer, Berlin (2006)

20. Kuratowski, C.: Sur les problèmes des courbes gauches en Topologie. Fundam. Math. 15, 271-283 (1930)

21. Rosenstiehl, P., Tarjan, R.E.: Rectilinear planar layouts and bipolar orientations of planar graphs. Discrete Comput. Geom. 1(4), 343-353 (1986) 